\title{
Eosinophilic gastroenteritis in a young man
}

\section{Giuseppe Famularo MD PhD, Cosimo Prantera MD, Josè Nunnari MD, Laura Gasbarrone MD}

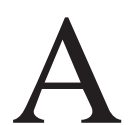

32-year-old man presented with a two-week history of lower abdominal pain, diarrhea without blood or mucus, and progressive abdominal swelling. He had no fever or recent weight loss, but he had experienced recurrent epigastric pain, nausea and episodic diarrhea for about one year. As a teenager, he had had tuberculous lymphadenitis. He reported not taking any illicit drugs, over-the-counter medications or herbal remedies. He had no personal or family history of gastrointestinal disorders or allergies.

On physical examination, the patient had a distended and diffusely tender abdomen with a shifting dullness. The rest of the examination was normal. His leukocyte count was 11.3 (normal $4-10$ ) $\times 10^{9} / \mathrm{L}$, with eosinophil levels of $8 \%$ to $27 \%$ (normal $\leq 7 \%$ ). He had an erythrocyte sedimentation rate of $120 \mathrm{~mm} / \mathrm{h}$, a C-reactive protein level of 150 (normal < 5) mg/L and a total IgE level of 612 (normal < 430) $\mu \mathrm{g} / \mathrm{L}$. The results of a peripheral blood smear and flow cytometry showed normal eosinophils and no blast cells or other atypical cells.

No ova or cysts were detected in microscopic examination of a stool specimen. The results of an enzyme-linked immunosorbent assay to detect parasite infestation, including species of Strongyloides and Toxocara, were negative. The results of skin prick tests for food and pollen allergies, screening for autoimmune disorders, and testing for antigliadin, antiendomysial and antitransglutaminase antibodies were also negative.

Electrocardiography and transthoracic echocardiography gave normal findings. Ultrasonography, computed tomography (Figure 1) and magnetic resonance imaging showed abundant ascites and diffuse thickening of the omentum. The upper and lower gastrointestinal tract appeared normal during endoscopic examination. However, microscopic examination of multiple biopsies showed mild to moderate villous atrophy without mucosal or submucosal inflammation, ulcers, granulomata or cryptitis. No eosinophilic infiltration was found in any of the specimens. Results of staining of the biopsy specimens for mycobacteria and fungi were negative, and culture grew no organisms.

A total of $1500 \mathrm{~mL}$ of ascitic fluid was removed by ultrasonography-guided paracentesis. It had a leukocyte count of $11.9 \times 10^{9} / \mathrm{L}$, with $87 \%$ eosinophils and no malignant cells. The results of culture and staining for bacteria, mycobacteria and fungi were negative. Because of worsening abdominal swelling and tenderness, we did an emergency laparoscopy with a midline incision. A large amount $(3000 \mathrm{~mL})$ of green-

\section{Key points}

- Symptoms of eosinophilic gastroenteritis depend on which layer of the bowel wall is involved by the eosinophil-rich infiltrate.

- Other disorders, such as drug hypersensitivity, infection, vasculitis or Crohn disease, should be excluded before the diagnosis of eosinophilic gastroenteritis is made.

- Although spontaneous remission may occur, some patients may require treatment with corticosteroids or other antiinflammatory agents.

- Outcomes are usually favourable; however, the long-term natural history of the disorder is unknown.

ish, turbid fluid was found in the peritoneal cavity, along with diffuse and nodular thickening of the peritoneum. Microscopic examination of a biopsy specimen of the peritoneum showed an extensive inflammatory infiltrate composed mostly of eosinophils, with sparse numbers of lymphocytes and macrophages (Figure 2). There was no evidence of vasculitis, thrombosis or viral cytopathic effect, nor were there granulomata, giant cells or intracellular parasites. The results of special staining for organisms were again negative.

Treatment with furosemide $(50 \mathrm{mg} / \mathrm{d})$ and spironolactone $(100 \mathrm{mg} / \mathrm{d})$ was started. Two weeks later, the patient was symptom free and ultrasonography showed no ascites. Diuretic treatment was gradually tapered and stopped after six weeks. When last seen, one year later, the patient was doing well. He had a normal eosinophil count and no evidence of ascites or active inflammatory disease.

\section{Discussion}

Eosinophilic gastroenteritis may involve any part of the gastrointestinal tract. An intense inflammatory infiltrate, consisting predominantly of eosinophils, macrophages and lymphocytes, penetrates into one or more layers of the gastrointestinal tract. ${ }^{1}$ In 1937, Kaijser described this disorder in two patients with syphilis who were allergic to neoarsphenamine; ${ }^{2}$ about 300 instances have been reported since then. ${ }^{1}$

From the Departments of Internal Medicine (Famularo, Gasbarrone), Gastroenterology (Prantera) and Pathology (Nunnari), San Camillo Hospital, Rome, Italy

CMAJ 2011. DOI:10.1503/cmaj.091467 


\section{Clinical, pathologic and endoscopic features}

Eosinophilic gastroenteritis is classified into mucosal, submucosal or muscular, and serosal types. ${ }^{1}$ Its clinical presentation, course and outcome depend on the location and depth of involvement of the different layers of the gastrointestinal tract. ${ }^{1}$ The inflammatory infiltrate is usually patchy in distribution and occasionally may be found in parts of the gastrointestinal tract that are macroscopically normal. ${ }^{1}$ In most instances, the endoscopic findings parallel the patchy distribution of the eosinophilic infiltrate and may vary from normal mucosa to mild erythema, thickened mucosal folds, nodularity and frank ulceration.
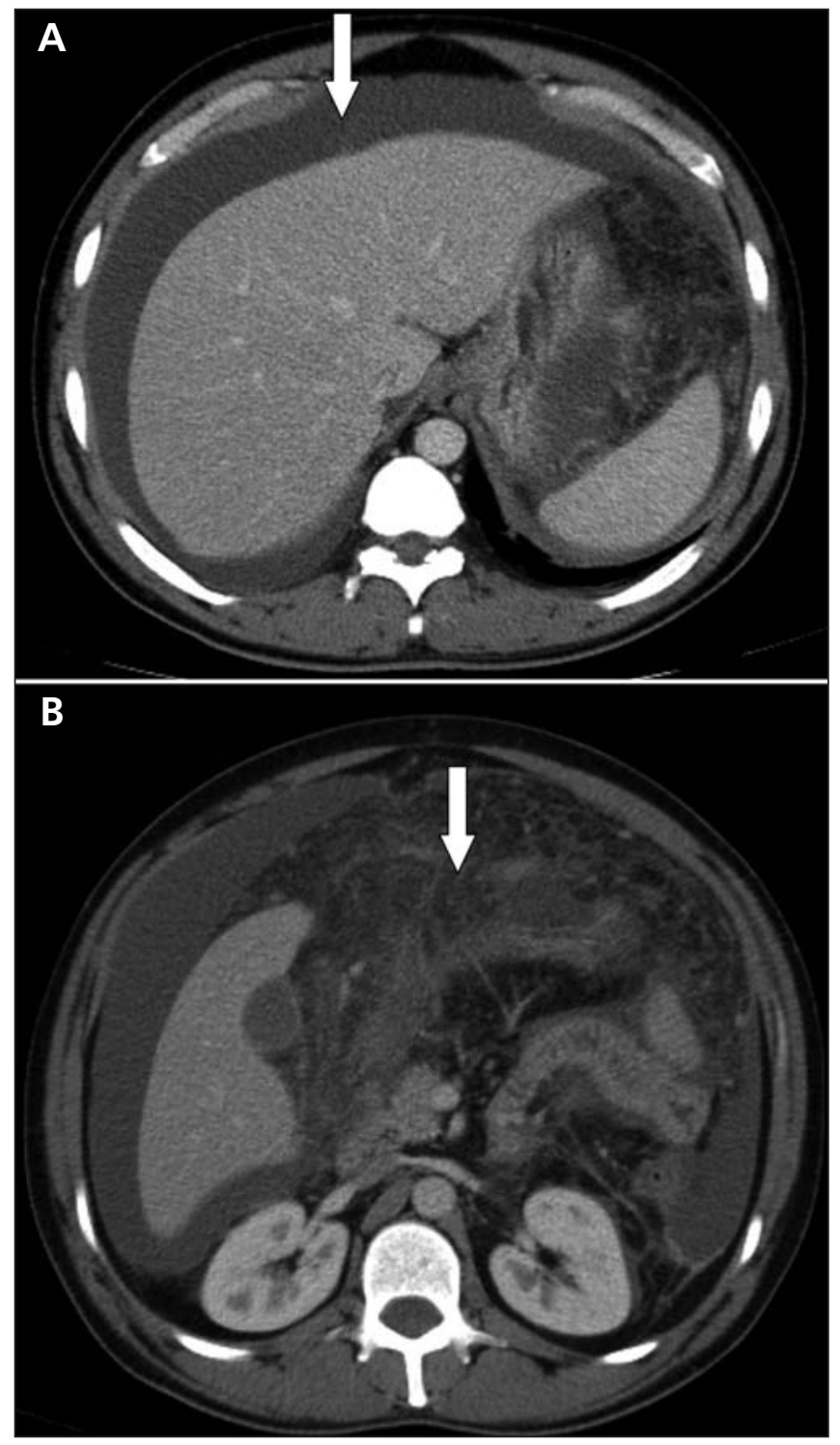

Figure 1: Computed tomography of the abdomen and pelvis of a 32-year-old man with eosinophilic gastroenteritis. (A) Axial images show a large volume of ascites in the perihepatic space (arrow). Abundant ascites was also present in the perisplenic and parietocolic spaces (not shown). (B) Stratification and increased enhancement of the mesentery and fat tissue of the small bowel (arrow) are present, which suggest serosal infiltration of the bowel wall and edema.
When eosinophilic gastroenteritis is suspected, multiple biopsy specimens should be taken to avoid missing the diagnosis of this misleading and potentially severe disorder. However, examination of mucosal specimens is frequently unrevealing when the eosinophil-rich inflammation involves only the deeper layers of the bowel wall. Mild to moderate villous atrophy may be present, which may confound the differential diagnosis with celiac disease or other malabsorption disorders. Pancreatitis, liver involvement and intussusception have been reported in some patients. ${ }^{1}$ The diagnosis may be delayed or missed in patients with milder forms of eosinophilic gastroenteritis, who often present with subtle and confounding clinical features.

Our patient had peripheral eosinophilia and eosinophilic ascites, which are the hallmarks of the serosal type of

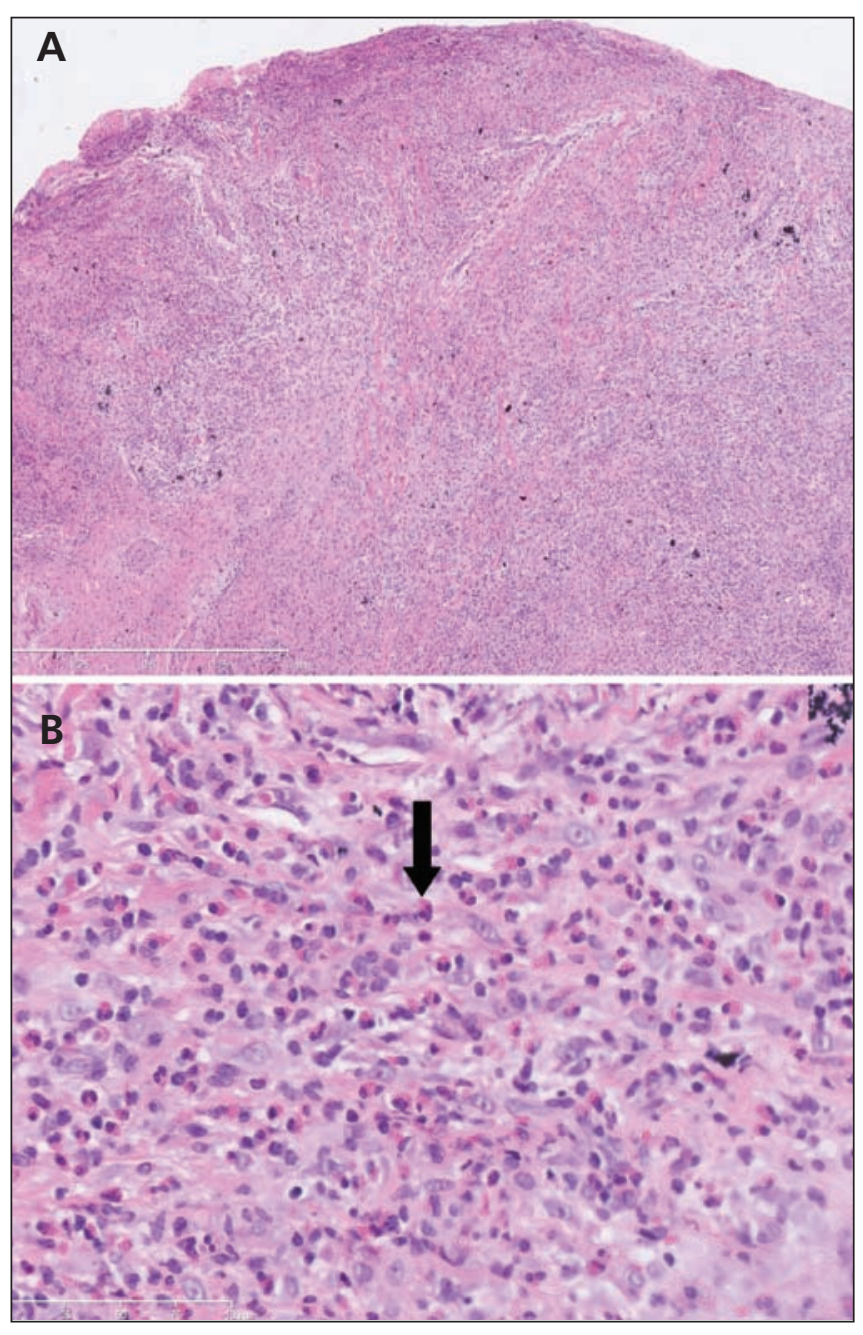

Figure 2: Full-thickness biopsy specimen of the patient's peritoneum. (A) A low-power view, showing extensive inflammatory infiltrate composed mostly of sheets of eosinophils, with sparse numbers of lymphocytes and macrophages, and thickened and edematous subserosa (hematoxylin and eosin stain, original magnification $\times 5$ ). (B) A higher-power view, showing numerous eosinophils (arrow), which are characteristically deep pink on staining with hematoxylin and eosin (original magnification $\times 40$ ). 
eosinophilic gastroenteritis. ${ }^{1}$ Our patient had no malabsorption, bowel obstruction or bleeding, which are typical features of the mucosal and muscular types. These findings highlight the point that only the peritoneum and the serosal layer of the gut may be involved, while both the mucosal and muscular layers are spared. We had to obtain full-thickness biopsy specimens for accurate diagnosis because the eosinophilic infiltrate was confined to the peritoneum and subserosal layer.

Patients with drug hypersensitivity, infection by a virus, Helicobacter pylori or a parasite, vasculitis (e.g., ChurgStrauss syndrome, polyarteritis nodosa), connective tissue disease (e.g., systemic sclerosis), celiac disease, Crohn disease, eosinophilic leukemia or hypereosinophilic syndrome may have eosinophilic infiltration of the gastrointestinal tract that could mimic the cardinal feature of primary eosinophilic gastroenteritis. ${ }^{1}$ These disorders should be ruled out before a firm diagnosis of primary eosinophilic gastroenteritis is established. Furthermore, involvement of organs other than the gastrointestinal tract usually points to systemic hypereosinophilic syndromes such as eosinophilic leukemia, hypereosinophilic syndrome and Churg-Strauss syndrome.

\section{Risk factors and pathophysiology}

Although the exact cause is unknown, an underlying food or drug allergy or an atopic disorder is found in up to $70 \%$ of patients, with most having increased total and food-specific IgE levels. ${ }^{1}$ In a review of 220 patients, up to $40 \%$ had a history of allergic bronchial asthma. ${ }^{3}$ Patients do not usually report a history of food-induced anaphylaxis, despite the common feature of food-specific IgE and atopy, ${ }^{1}$ which suggests that tissue injury is triggered by cell-mediated immunity and eosinophil activation rather than IgE hypersensitivity. Except for atopy, no other risk factors for eosinophilic gastroenteritis are known.

The basic pathophysiologic defect of eosinophilic gastroenteritis is unknown, and the role of food allergies remains controversial. Altered mucosal integrity and impaired function of the epithelial barrier are believed to facilitate the translocation of luminal bacteria and food products. ${ }^{4}$ Localization of these substances across the gastrointestinal wall or in the peritoneum is thought to elicit an allergy-like inflammatory response with eosinophilic infiltration. If unchecked, this response could damage the epithelial barrier further and amplify both translocation and inflammation. Tissue injury is ultimately induced by basic proteins, including the eosinophil major basic protein, cationic protein and peroxidase, which are released from eosinophils. ${ }^{4}$

The association between eosinophilic gastroenteritis and other idiopathic eosinophilic disorders of the gastrointestinal tract, such as eosinophilic esophagitis and eosinophilic colitis, is unclear. These disorders likely represent a continuum of pathologically and mechanistically related disorders that have in common the involvement and activation of eosinophils as the ultimate effector of injury to the gastrointestinal tract. ${ }^{4}$ This hypothesis is still unproven and requires further investigation.

\section{Treatment, prognosis and complications}

The severity of eosinophilic gastroenteritis may wax and wane. Spontaneous recovery has been reported in some patients, including those with the serosal type of the disorder. ${ }^{1}$ Because no recurrence of ascites was observed in our patient after the peritoneal fluid was removed, we decided not to start treatment with corticosteroids or other antiinflammatory medications. Watchful observation confirmed that the patient achieved a full and complete recovery, and this spontaneous remission was maintained after more than one year of follow-up.

In most patients, the response to corticosteroids is rapid; however, long-term treatment with corticosteroids, immunosuppressants, sodium cromoglycate or leukotriene-receptor antagonists may be needed.

Deaths are uncommon and are usually due to perforation of the gastrointestinal tract. ${ }^{1}$ In most patients, the disorder is benign, but its natural history is unknown, which indicates the need for close follow-up of patients over a long time. In rare cases, the disorder may be the initial manifestation of a progressive, multiorgan hypereosinophilic syndrome. A clonal bone marrow disorder such as acute or chronic eosinophilic leukemia with gastrointestinal involvement may develop in some patients. 5

\section{Competing interests: None declared.}

Contributors: All of the authors participated equally in analyzing and interpreting the data, writing the article and revising it critically for important intellectual content. All of the authors approved the final version submitted for publication.

Acknowledgements: The authors thank Drs. Michele Galluzzo and Daniele Remotti for their helpful comments and critique of earlier versions of this paper.

\section{REFERENCES}

1. Khan S, Orenstein SR. Eosinophilic gastroenteritis. Gastroenterol Clin North Am 2008;37:333-48

2. Kaijser R. Zur Kenntnis der allergischen Affektionen des Verdawungskanal vom standpunkt desi Chirugen aus. Arch Klin Chir 1937;188:36-64.

3. Naylor AR. Eosinophilic gastroenteritis. Scott Med J 1990;35:163-5.

4. Yan BM, Shaffer EA. Primary eosinophilic disorders of the gastrointestinal tract. Gut 2009;58:721-32.

5. Martinelli G, Rondoni M, Ottaviani E, et al. Hypereosinophilic syndrome and molecularly targeted therapy. Semin Hematol 2007;44(Suppl 2):S4-S16.

Correspondence to: Dr. Giuseppe Famularo, Department of Internal Medicine, San Camillo Hospital, Circonvallazione Gianicolense, 00152 Rome, Italy; gfamularo@scamilloforlanini.rm.it

The section Cases presents brief case reports that convey clear, practical lessons. Preference is given to common presentations of important rare conditions, and important unusual presentations of common problems. Articles start with a case presentation (500 words maximum), and a discussion of the underlying condition follows (1000 words maximum). Generally, up to five references are permitted and visual elements (e.g., tables of the differential diagnosis, clinical features or diagnostic approach) are encouraged. Written consent from patients for publication of their story is a necessity and should accompany submissions. See information for authors at www.cmaj.ca. 